\title{
Teaching old drugs new tricks to treat COVID-19
}

In early 2020, scientists and medics started rummaging around the pharmacy shelves hunting for treatments that might curtail the spiralling pandemic death rates. This is the story of how they are searching and what they have found so far.

\section{Nina Notman}

$\mathrm{R}$ emdesivir's story began back in 2009 when Gilead drug hunters were searching for an antiviral drug to treat hepatitis $\mathrm{C}$ and respiratory syncytial virus ${ }^{1}$. Despite recognizing the molecule's ability to interrupt viral replication, clinical trials against these viruses never happened. It was eventually dosed in humans in 2015, after it was pivoted towards Ebola during a large outbreak in West Africa. However, research into remdesivir against this virus also ceased a few years later when two monoclonal antibodies were shown to be more effective.

Gilead scientists remained optimistic about remdesivir's potential. "We continued to explore the broad spectrum activity of remdesivir in animal models of inhibiting emerging viruses and in particular the early coronaviruses," says Richard Mackman, vice president of medicinal chemistry at Gilead Sciences. "It made sense based on that body of data to move forward in the clinic for COVID-19."

The first COVID-19 patient received remdesivir in late January 2020. “That was in response to a compassionate request for one of the first identified [COVID-19] patients in the US," says Anu Osinusi, vice president of virology at Gilead Sciences. More than 50 countries have now approved - either conditionally or fully - remdesivir for patients with severe COVID-19.

\section{Discovering new uses}

The idea of designing a medicine for one disease then pivoting or expanding its use to another isn't new. Typically, a novel treatment takes over a decade and US\$1.3 billion to reach the bedside ${ }^{2}$. Repurposing an already approved drug, or even an experimental drug, can potentially save on both of these.

"It's been through all of the preclinical work needed to get approval for an in-human clinical trial and it's been in humans so we have an understanding of the pharmacokinetics and the safety," says Matt Hall, director of the early translation branch at the US National Institutes of Health's National Center for Advancing Translational

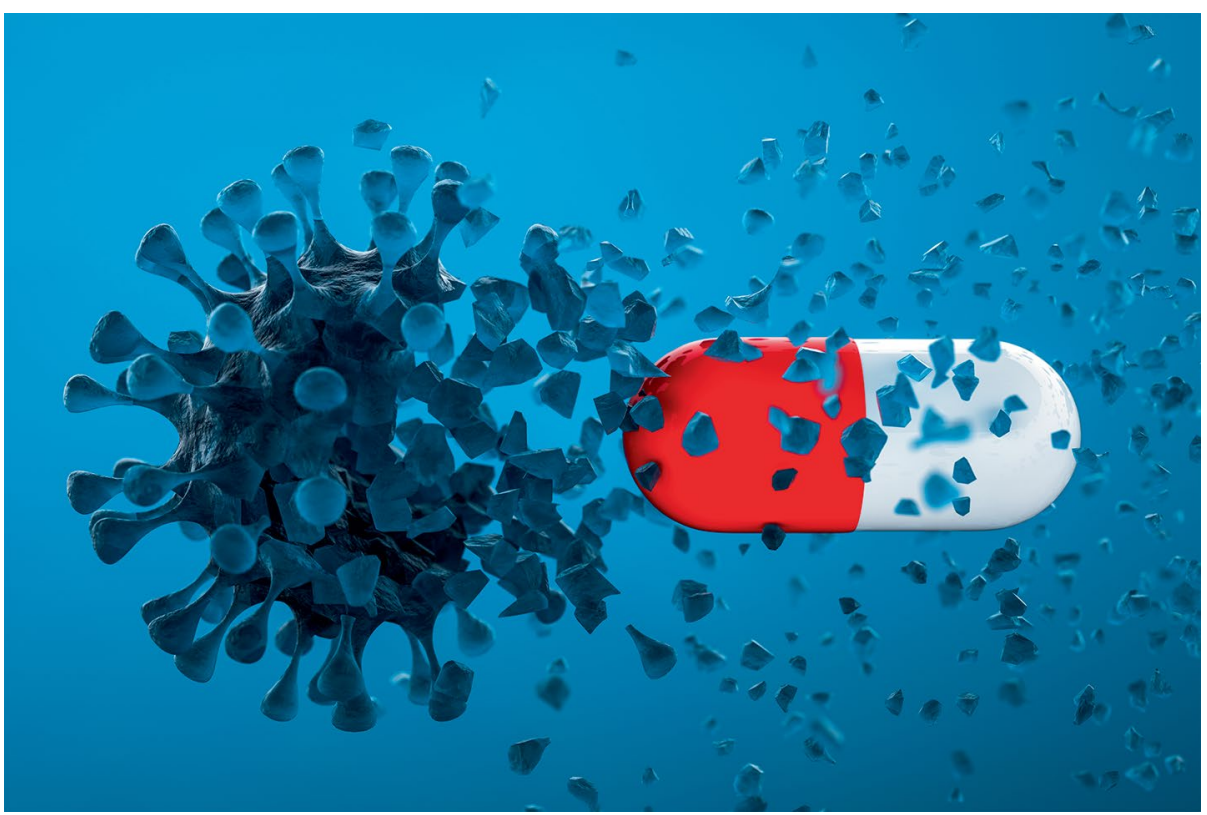

Credit: Dowell / Moment / Getty

Sciences. "You've removed most of the timeline to get to a clinical trial and obviously the cost is significantly reduced," he adds.

Pre-clearing the early hurdles on the drug development pathway enhances the chances of success as well. "The advantage of drug repurposing is that you are eliminating some of the risk," explains Don Frail, a San Francisco-based former pharma executive now starting and advising early biotechs; he previously managed teams exploring repurposing opportunities at both Pfizer and AstraZeneca.

\section{Repurposing during a pandemic} Nevertheless, the chance of failure remains high. The biggest remaining challenge is proving efficacy - how well the drug performs against the new indication. Around three quarters of all drugs won't make it through phase II efficacy trials and another one in three won't get to phase III, even when they have been designed with a specific indication in mind ${ }^{3}$.
The UK National Health Service's RECOVERY trial, which started in early 2020, quickly dispelled hopes that the antimalaria medication hydroxychloroquine - as well as the antibiotic azithromycin and a few other authorized medicines - could be useful against patients hospitalized with COVID-19.

RECOVERY is the largest clinical trial testing repurposing drugs for severe COVID-19, with over 45,000 patients enrolled by late November 2021 . There have been some significant successes; including the steroid dexamethasone, monoclonal antibody tocilizumab and Regeneron's investigational monoclonal antibody combination. Trials are still being conducted on the rheumatoid arthritis treatment baricitinib, and the psoriasis and multiple sclerosis drug dimethyl fumarate.

Additionally, thousands of smaller repurposing trials are underway for treating both hospitalized and non-hospitalized patients. Among these, the UK PRINCIPLE trial and the NIH ACTIV-6 trial are 


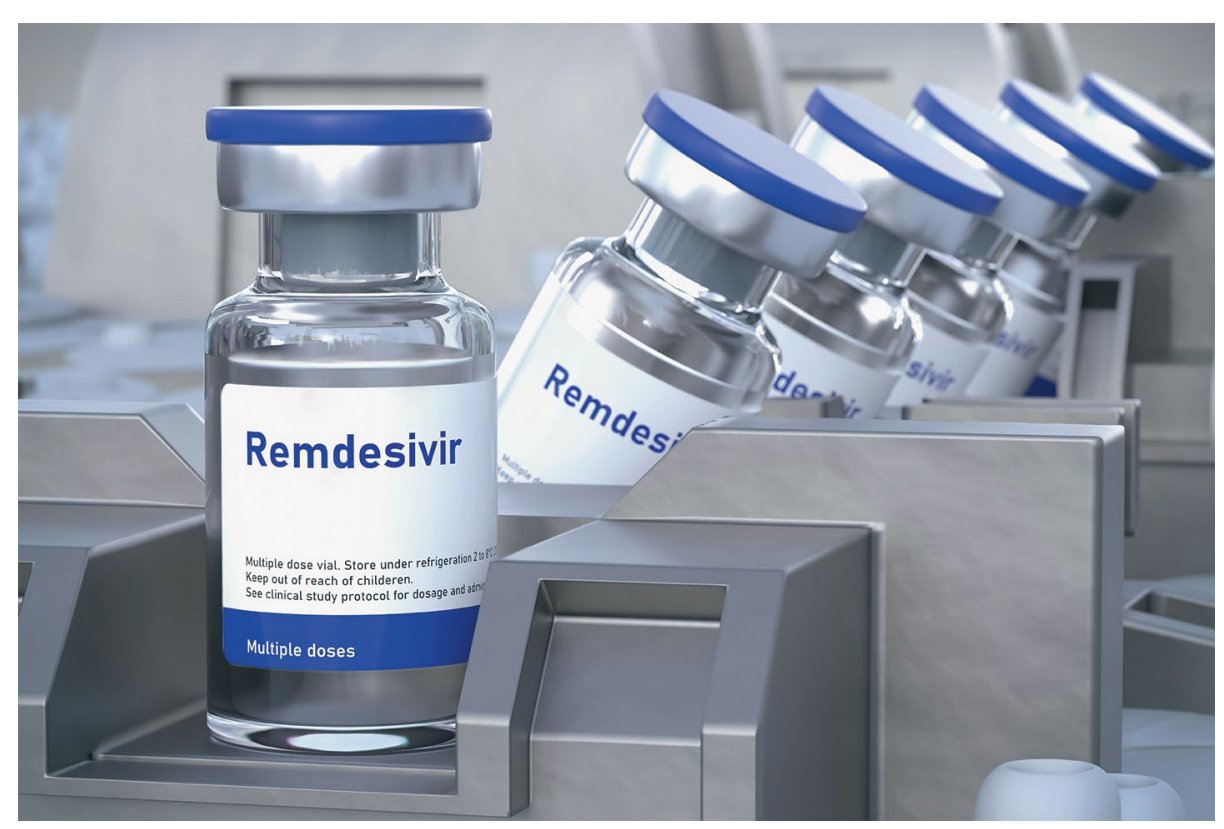

Credit: Monika Wisniewska / Alamy Stock Photo

the most significant. The PRINCIPLE study has already enrolled nearly 7,000 participants and given the inhaled steroid budesonide the thumbs up. It continues to assess other therapeutics including the flu drug favipiravir. Meanwhile, ACTIV-6 is examining the antidepressant fluvoxamine and the steroid combination fluticasone. "One of the key features of ACTIV-6 is that these are medications that can be taken in a pill, they can be taken at home," says Hall.

\section{A needle in a hay stack}

Approximately 20,000 prescription drugs are FDA-approved in the US. Many thousands of other current and previous drug candidates have also been administered safely to humans but haven't - or at least not yet - made it to market. These numbers raise the question: where does one start when hunting for an old drug for a new use?

An unexpected clinical observation may be the best place. Viagra is the most famous example of this; originally intended for high blood pressure and angina, Pfizer switched the indication for its little blue pill to erectile dysfunction after clinical trial participants reported an unanticipated side effect. Indications known to have similar disease pathways are another obvious launching point - and this is why some oncology drugs gain approval for one cancer, then another, then another.

There was never a clear-cut first-line drug for COVID-19. No one has developed a drug for treating severe coronavirus before - they are normally fairly innocuous viruses with over-the-counter medications, such as painkillers and decongestants, providing adequate symptom relief. Remdesivir, with its limited preclinical data against other coronaviruses, was one of the few options on the table. However, since remdesivir hadn't been approved for an indication prior to COVID-19, only a few people had access to it during the early days of the pandemic. Even now, the use of remdesivir remains limited by its high price and ongoing concerns about its efficacy.

In October 2021, promising trial results for another antiviral - molnupiravir - were published. Merck, which is championing this oral therapeutic, says it reduced hospitalizations and deaths by half in trial patients who received it. Molnupiravir, unlike remdesivir, was never previously tested in humans for another indication. However, it had been tested on animals against a number of viruses, including coronaviruses. On 4 November 2021, the UK approved molnupiravir for use in patients with mild to moderate COVID19 and at least one risk factor that might contribute to severe illness.

\section{Inventory assistance}

In early 2020, with no obvious go-to treatment available, medics around the world began searching for a COVID-19 cure in their medicine cabinets. "Over 100 different drugs were used in the first three months of pandemic," says David Fajgenbaum, an immunologist at the University of Pennsylvania in Philadelphia.

Fajgenbaum first came across the concept of drug repurposing at medical school, when he was searching for a cure for his own life-threatening illness, the rare inflammatory disease Castleman disease. Having failed to respond to approved treatment options, he began treating himself off-label with drugs already approved for indications with similar disease pathways to Castleman disease. He has been in remission for seven years and counting thanks to the immunosuppressant sirolimus, approved for kidney transplant patients to reduce the likelihood of organ rejection. "We've now successfully identified multiple drugs for Castleman's that were already approved for other things," Fajgenbaum says.

When COVID-19 arrived, Fajgenbaum realized his previous experience of drug repurposing could be useful. He created a database, CORONA, which synthesizes all the published data on drugs tested against COVID-19, ranging from individual case studies to full clinical trials. Currently, CORONA has data on over 400 medications given to more than 300,000 patients, he says. Clinical trial planners and funders are the intended end users of the inventory. "The CORONA project data was utilized for ACTIV-6 to pick which drugs to include in the trial," says Fajgenbaum.

\section{Back to basics}

Boosting understanding of the disease pathways was another way to direct the selection of drugs to be trialled against COVID-19. In early 2020, Nevan Krogan, a systems biologist at the University of California, San Francisco, realized that his research could help with this.

Krogan's bread and butter is identifying human proteins that are affected by diseases. "Viruses have a limited genomic capacity just 15 to 30 genes compared to 20,000 in us. They need our genes and proteins to live and replicate and infect our cells," he says. This information can then be used to develop therapeutics that inhibit the viruses from infecting human cells. (A more common approach to drug development is to focus on disarming the virus itself.)

The Krogan lab tagged each of the SARS-CoV-2 proteins with a short peptide sequence before introducing the virus to human cells. The scientists then separated the proteins, with the help of the peptide tag, and used mass spectrometry to identify which human proteins were physically connected to which viral proteins. They combined this information with computational analysis to create a protein interaction map; a blueprint of more than 300 human proteins that assist SARS-CoV-2 as it infects and then replicates in the body ${ }^{4}$. "We have done this for many pathogens and diseases including breast cancer and 


\section{Box 1| Al and drug manufacture}

When an old drug gets given a new life against a disease that is killing thousands of people every day, another challenge arises - how to rapidly manufacture enough of it to meet global demand.

In March 2020, multiple supply chain issues were emerging. "Things like macaroni and cheese and toilet paper were disappearing from the shelves," says Tim Cernak, a medicinal chemist at the University of Michigan, Ann Arbor. The chemical supplier MilliporeSigma also began noticing that it was being bought out of the starting materials for key potential treatments for COVID-19, he adds.

This saw an existing collaboration between Cernak and MilliporeSigma pivot towards COVID-19. "We had been working with them for some time on exploring a retrosynthetic software, called SYNTHIA, that attempts to logically disconnect target molecules into pieces you can buy," says Cernak. There are nearly 70 million chemical starting materials on the market and an

head and neck cancer over the years," says Krogan.

Brian Shoichet, a pharmaceutical chemist also at the University of California, San Francisco, and other collaborators then helped Krogan search through drug and drug candidate inventories for likely targets. "We looked at the human targets and asked are there any drugs that are known, or likely, to modulate the human targets that the virus is subverting," says Shoichet. They identified 69 molecules and used assays to check their antiviral activity. Two drug categories jumped out, protein translation inhibitors and Sigma receptors such as hydroxychloroquine ${ }^{5}$. The latter turned out to be a wild goose chase ${ }^{6}$, but the former is still looking hopeful.

Several drug candidates, originally developed as anticancer agents, are entering clinical trials for COVID-19 as a direct result of this research. PharmaMar's plitidepsin, a natural product from sea squirts, is currently in phase III and eFFECTOR Therapeutics's Zotatifin is in phase Ib. "There's a bunch of other clinical trials ongoing, based on our maps, that we're just peripherally following too," adds Krogan.

\section{Screening at scale}

In the case above, the team tested a carefully selected group of drugs and drug candidates for antiviral activity against representative biological assays. A more common approach ever-increasing number of possible chemical reactions. "The computer can handle that amount of data a lot better than the human can," he adds.

SYNTHIA was used by his team to discover alternative pharmaceutical building blocks and devise new synthetic routes for a dozen drugs being considered for treating COVID-19. These included remdesivir, umifenovir and baricitinib. "We were asking if there was a way that we can provide more recipes for making these drugs should they be needed," says Cernak. A number of the identified routes were shorter and with cheaper starting materials than those currently used. With one drug - bromhexine - made in his lab in a single step.

Drug manufacture is a highly regulated industry, and changing synthetic routes involves a lot of hoop jumping. Thus far, Cernak's ideas have not had to be implemented because the drug supply chain has generally held up to the challenge posed by the pandemic.

is to screen a library of hundreds, if not thousands, of drug molecules against these types of assays. This process is called high-throughput screening.

The California Institute for Biomedical Research (Calibr), the drug discovery arm of Scripps Research, in San Diego holds a physical library of over 12,000 active drug ingredients for this purpose: the ReFRAME library. These include both approved drugs and drug candidates. "The purpose of it was to come up with a comprehensive drug repurposing collection that we could use for a variety of screens primarily in neglected tropical diseases," says Arnab Chatterjee, a medicinal chemist at Calibr.

About half of the molecules in the ReFRAME library are commercially available, the others are synthesised in-house. "We make the collection available free of charge to anyone in the world who wants to use the collection for screening," says Chatterjee. The team recognized the collection's potential for helping during the pandemic in very early 2020, and began rapidly shipping its library to researchers in other labs in the US and beyond for testing.

This idea of screening massive drug and drug candidate libraries in search for repurposing opportunities is not without its critics. "This belief that by screening a ton of drug molecules against a laboratory assay without a mechanistic hypothesis will lead to an aha moment and cure the world of whatever the disease of the day is has been going for 20 years," says Aled Edwards, a biochemist at the University of Toronto.

Nevertheless, so far, this approach hasn't yet led to any actual drugs being approved for new indications, he adds. "There's nothing inherently flawed about the concept, it just hasn't worked out." The team Frail led at Pfizer stopped chasing this approach early on. "We pursued it and concluded that the hit rate wasn't high enough, relative to the investment required, to continue," he says. While the overall number of hits were low, some very novel biology was discovered during this type of screening, he adds, so the work wasn't entirely in vain.

Chatterjee agrees that this scattergun approach is not very likely to pinpoint a perfect drug for a new indication. Instead, it offers a chemical starting point from which to develop a novel drug molecule, he says. "We can take a hit structure that we know a lot about, because it's a clinical compound and it's been in humans, and then develop a more potent and safer molecule based on what we know about its track record," Chatterjee adds. His lab is currently using results from the screenings of the ReFRAME collection to develop a novel coronavirus drug.

\section{Data acquisition}

The NIH National Centre for Advancing Translational Science also has a physical library of active drug ingredients compiled for exploring repurposing opportunities. "There are about 3,000 molecules in our approved drug library and another over 5,000 molecules that have either been in clinical trials or in development for clinical trials," says Hall.

The NIH drug library, assembled originally for rare diseases, was redirected towards COVID-19 when the pandemic hit. "We designed 30 assays for COVID-19 from biochemical assays, to those containing viral proteins and then also infection assays including the live virus," Hall says. His team tested the drug library against these assays and rapidly posted the data on a public portal for others to use. "We wanted to make it available to the AI community so they could use it to develop in silico methodologies for predicting treatments for patients," he explains.

Over the past five years or so, the use of artificial intelligence (AI) in the pharmaceutical industry has exploded. Increased computational prowess and much more data being openly available enabled this surge, says Feixiong Cheng, a network medicine researcher at the Cleveland Clinic's Genomic Medicine Institute. "We really need big data to tune the AI and deep learning models," he says. 
Using far more data than humans are capable of, AI can spot subtle or complex patterns humans might not have noticed. This means it offers valuable insight at many stages during the drug discovery process, including identifying initial hits, optimizing leads, directing pre-clinical research, and selecting patients for clinical trials. It can even support synthetic route planning (see Box 1).

\section{A wider net}

Many AI companies looked for new ways to use their AI toolkit to help at the start of the pandemic. BenevolentAI, for example, went hunting for drug-repurposing opportunities. With over a billion known and assumed connections between diseases, symptoms, genes, and proteins within its knowledge graph, created by pulling data from journal articles and patents, the London-headquartered AI-driven drug discovery company normally focuses on developing drugs from scratch. Its scientists had however worked on drug repurposing once before, finding a new drug combination for treating a rare childhood brain cancer.

BenevolentAI's data scientist Dan Smith and his team began work on COVID-19 in January 2020. The company's knowledge graph first needed updating to include more viral biology. "We used AI to rapidly enhance our knowledge graph with COVID19 related information and pulled out 40,000 new relationships from the literature, for our algorithms and tools to use, in a matter of hours," says Smith. The scientists then asked their system questions such as which are the overarching biological pathways involved in SARS-CoV-2 infecting the lungs and which gene proteins are involved. "Our scientists are able to form and answer complex questions, by traversing our knowledge graph to find things of interest," Smith says.

The team then enhanced the network by adding other relevant biological contexts, so that it eventually represented, at a molecular level, both how SARS-CoV-2 enters and infects the body, as well as the downstream inflammatory response. "The final result was a network of about 600 gene proteins with two modules really standing out," says Smith. The AI found 40 drugs and drug candidates that work on these two areas of biology and further filtering led to the
Janus kinase (JAK) inhibitor baricitinib. The whole process took two days. The team then informed Eli Lilly of its findings, the drug owner was previously unaware of its antiviral properties. In November 2020, following clinical trials, the FDA approved the use of baricitinib, in combination with remdesivir, for ventilated COVID-19 patients. The UK RECOVERY trial also includes baricitinib.

\section{A virtual treasure trove}

AI-driven drug discovery and development firm, Healx, in Cambridge, UK, has also applied its AI methods to COVID-19. David Brown, who co-created Viagra while at Pfizer, co-founded Healx with the aim of redeveloping existing drugs to treat rare diseases. "We have ten different [AI] methods that look for connections between drugs and diseases," says Neil Thompson, the Healx chief scientific officer. More often than not, the search is not for one drug, but for a combination.

"We look at the fingerprint of the disease versus normal, and then look for drugs that reverse that fingerprint, squashing what's over-expressed down to normal or increasing expression of what's under-expressed back to normal," says Brown. "We build drug combinations in a very rational way, looking for synergy of the positive effects and avoiding the negative effects," he adds. Regarding what research Healx is doing around COVID-19, the company is tight-lipped, other than to say it is currently evaluating the results of its predictions in preclinical assays.

Meanwhile, Biovista, a Charlottesville biotech company that also employs AI to repurpose drugs, has set its sights on longterm health outcomes from COVID-19. A growing number of COVID-19 patients are reporting long-term symptoms, including fatigue, breathlessness, headaches and cough. "There are approximately 98 of these complications and counting, but each of the long COVID complications is [an already known] standalone disease," says Biovista co-founder Aris Persidis. By using AI to map drugs and drug candidates against known clinical outcomes, Biovista has been unravelling biological links between COVID-19 and known diseases, and thereby identify potentially useful therapeutics.
As with Healx, Biovista isn't revealing what it found just yet. In June 2021, however, the Cleveland Clinic's Cheng reported he had used AI to help identify the mechanisms by which COVID-19 can lead to Alzheimer'slike dementia ${ }^{7}$.

\section{Collaboration for the win}

One of the few highlights of the pandemic has been watching the scientific and medical communities work together to identify repurposable drugs - and develop vaccines - to combat COVID-19. It has been a powerful demonstration of what is possible when everyone pulls together towards a common goal. But has anything been learned from the pandemic that will change the long and arduous process of developing drugs for other diseases in the future?

Only if we do not go back to our old ways, says Krogan. "We've shown how fast we can move when the scientific community - the institutions, the pharmaceutical companies and academia - all work together." The inherent competitiveness of academic science and the need for companies to turn a profit has historically led most drug developers to work in secretive silos. "How fast we can move when we break down these silos was showcased during this pandemic and now the challenge is to keep the spirit alive so that we're ready for the next pandemic." Other complex diseases with limited treatment options such as some cancers and Alzheimer's should also be studied like this, he adds. "If we did, we would have a lot more treatments for a lot more diseases."

\section{Nina Notman $\bowtie$ \\ Freelance science writer, Salisbury, UK. \\ Twitter:@ninanotman

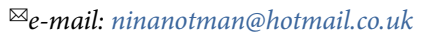

\author{
Published online: 12 January 2022 \\ https://doi.org/10.1038/s44160-021-00013-0 \\ References \\ 1. Eastman, R. T. et al. ACS Cent. Sci. 6, 672-683 (2020). \\ 2. Wouters, O. J., McKee, M. \& Luyten, J. JAMA 323, 844-853 (2020). \\ 3. Dowden, H. \& Munro, J. Nat. Rev. Drug Discov. 18, 495-496 (2019). \\ 4. Gordon, D. E. et al. Science 370, eabe9403 (2020). \\ 5. Gordon, D. E. et al. Nature 583, 459-468 (2020). \\ 6. Tummino, T. A. et al. Science 373, 541-547 (2021). \\ 7. Zhou, Y. et al. Alzheimer's Res. Ther. 13, 110 (2021). \\ 8. Lin, Y. et al. Nat. Commun. 12, 7327 (2021).
}

